\title{
Comparing Dimensions of Four-Strand Hamstring Tendon Grafts with Native Anterior and Posterior Cruciate Ligaments
}

\author{
Barış Yılmaz, ${ }^{1}$ Güzelali Özdemir, ${ }^{2}$ Elif N. Keskinöz, ${ }^{3}$ Gamze Tümentemur, ${ }^{3}$ \\ Kemal Gökkuş, ${ }^{4}$ and Bahtiyar Demiralp ${ }^{5}$ \\ ${ }^{1}$ Fatih Sultan Mehmet Education and Research Hospital, Department of Orthopedic Surgery and Traumatology, Istanbul, Turkey \\ ${ }^{2}$ Ankara Numune Education and Research Hospital, Department of Orthopedic Surgery and Traumatology, Ankara, Turkey \\ ${ }^{3}$ Department of Anatomy, Acıbadem University, Istanbul, Turkey \\ ${ }^{4}$ Memorial Antalya Hospital, Orthopedics and Trauma Department, Antalya, Turkey \\ ${ }^{5}$ Medipol University Hospital, Department of Orthopedic Surgery and Traumatology, Istanbul, Turkey
}

Correspondence should be addressed to Barış Yılmaz; drbyilmaz@yahoo.com

Received 25 August 2016; Revised 29 November 2016; Accepted 30 November 2016

Academic Editor: Adrian Dragu

Copyright (C) 2016 Barıș Yılmaz et al. This is an open access article distributed under the Creative Commons Attribution License, which permits unrestricted use, distribution, and reproduction in any medium, provided the original work is properly cited.

\begin{abstract}
Background. The aim of the study was to evaluate whether or not there was any incompatibility between four-strand hamstring tendons taken from the same knee and the dimensions of the ACL and PCL. Methods. 15 fresh frozen cadaver hamstrings were prepared as four-strand grafts and measurements made of the ACL and PCL circumferences in the midsection were made in the narrowest part of the midsection. The cross-section areas and diameters were calculated with geometric calculations used to measure the cross-sectional area of cylinders. Accepting that the geometric insertions were elliptical, the length, width, and area were calculated for entry areas. Results. A significant relationship at $96.2 \%$ was determined between the ACL mid and the hamstring diameter. A significant relationship at $96.7 \%$ was determined between the ACL and the hamstring mid area. A significant relationship at $96.4 \%$ was determined between the PCL mid and the hamstring diameter. A significant relationship at $95.7 \%$ was determined between the PCL and the hamstring mid area. Conclusion. For the reconstruction of ACL and PCL, it was determined that there is less incompatibility between the four-strand hamstring tendons taken from the same knee and the dimensions of the midsection PCL compared to the ACL dimensions.
\end{abstract}

\section{Introduction}

The bone structure which forms the knee joint is a stable structure due to the ligaments of the knee. The two important ligaments providing this stability are the anterior cruciate ligament (ACL) and the posterior cruciate ligament (PCL), the anatomy of which are becoming increasingly known [1-4]. As a result of studies which have focused on the equivalence of the ACL and PCL projections, there has been extensive research on the anatomic dimensions of the ACL and PCL and variations have been determined in various individuals [5].

The use of a hamstring tendon autograft is a widely preferred method in the treatment of injuries of these ligaments. Some previous anatomic studies have been conducted to evaluate the appropriate dimensions of hamstring tendons compared with the natural ACL, particularly in the form of the midsection and the entry area [6]. However, to the best of our knowledge, there has not yet been any study related to the PCL.

In one such previous study, it was reported that increased graft size did not improve the time zero biomechanical stability of the knee after ACL reconstruction (ACLR). It is found that increased graft size did not compensate for biomechanical instability resulting in a nonanatomic tunnel position [7]. There are also the potential risks that an oversized graft will disturb the healing process and kinematics [6]. Using arthroscopic second looks, Marzo et al. and Toritsuka et al. demonstrated that graft deterioration occurred mainly in the midsubstance zone $[8,9]$. Thus size matching of 
graft midsubstance with native ACL and PCL becomes increasingly important assuming anatomic tunnel position restoration. Therefore, the compatibility of the dimensions of four-strand hamstring tendon grafts with native ACL and PCL will provide additional knowledge for the application of hamstring autograft.

The aim of this study was to evaluate whether or not there was any incompatibility between four-strand hamstring tendons taken from the same knee and the dimensions of the ACL and particularly the PCL, which has not been previously investigated.

\section{Material and Methods}

In this anatomic study, evaluation was made of 15 knees of fresh frozen cadavers which had been stored at $+4^{\circ} \mathrm{C}$. The cadavers were $6(60 \%)$ males and $4(40 \%)$ females. The knees used were 5 right side and 4 left side from the male cadavers and 3 right and 3 left from the female cadavers. Exclusion criteria were knees with evident osteoarthritis (>Outerbridge Grade 3), those with osteophytes on the intercondylar notch (femur and/or tibia), or those with torn ACL or PCL or damaged hamstring tendons. None of the knees which were included for evaluation had any previous surgical scar or any sign of clinical deformation. The age of the cadavers at death was 51, 60, 63, 64, 69, 76, 78, 81, 82, and 94 years, respectively, and mean BMI was $25.2 \pm 5.76 \mathrm{~kg} / \mathrm{m}^{2}$ (range, $\left.18-34 \mathrm{~kg} / \mathrm{m}^{2}\right)$. The mean time from death to dissection was 1 month (range 0-3 months). The knee movements measured with a goniometer showed minimum $120^{\circ}$ flexion and full extension.

To harvest the hamstring grafts, a standard open anteromedial approach was used. The gracilis and semitendinosus tendons were identified in the tibial entry region and after the standard opening procedure, the grafts were taken separately using a closed stripper. Then the grafts were prepared and cleaned. Manual measurements were taken of the surroundings of the four-strand tendons with a digital goniometer.

The knee joint was opened for later removal of the ACL and PCL. Before taking the measurements, the soft tissues including the synovial membrane were carefully removed. The manual measurements of the ACL and PCL circumference were taken at approximately $1.5 \mathrm{~cm}$ proximal to the tibial entry area, which is the narrowest part of the midsection [6] (Figure 1). To preserve the natural tension of the graft, these measurements were made before cutting the ACL and PCL. When taking the measurements, first the ACL was measured; then to be able to measure the PCL, the ACL was cut and cleaned. At the same time, to be able to better expose the posterior region of the PCL, the structures in the posterior of the knee joint were carefully cleaned. Thus by fully exposing the PCL, the measurements were taken. The ACL and PCL were then completely removed. The cross-sectional areas and diameters were calculated with the geometric calculations which are used to measure the cross-sectional area of a cylinder. As the ACL and PCL resemble simple cylinders, the midsection was used to estimate the smallest cross-sectional area in both the ACL and PCL. The femoral and tibial entry

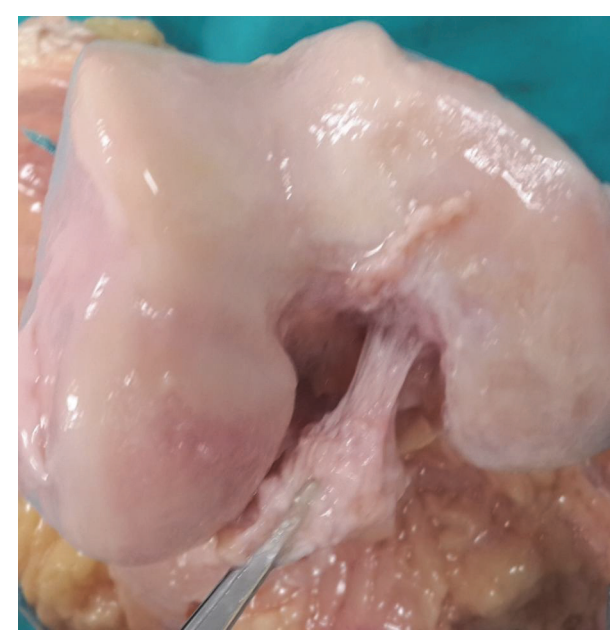

FIGURE 1: PCL circumference measurements made in the midsection $1.5 \mathrm{~cm}$ proximal to the tibial entry area after removal of the synovial membrane.

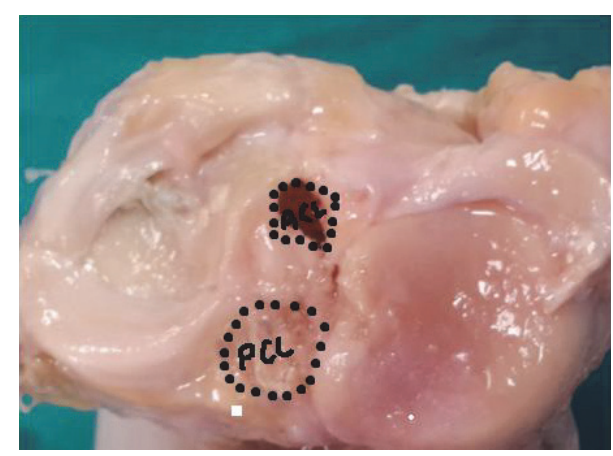

FIGURE 2: Measurements of the surface area of the tibial entry area without the synovial membrane.

areas of the ACL and PCL were then measured. Accepting that the insertions were geometrically elliptical, calculations were made of the length, width, and areas for both the ACL and the PCL. The length measurements were taken from the major axis of the entry areas and the width was measured perpendicular to the long axis at the widest point [6] (Figures 2 and 3 ).

Statistical analyses of the findings obtained in the study were made using IBM SPSS v. 22 (IBM, SPSS, Turkey) software. When evaluating the study data, conformity to normal distribution was tested with the Shapiro Wilk test. In the evaluation of the relationship between parameters, Pearson correlation analysis was applied in addition to descriptive statistics. A value of $p<0.05$ was accepted as statistically significant.

\section{Results}

All the results are summarised in Tables 1 and 2 .

A correlation was determined between the hamstring tendon dimensions and the ACL and PCL (diameter, crosssection area, $p<0.01)$. The mean diameter of the hamstring 
TABLE 1: Summary of results.

\begin{tabular}{lccccc}
\hline Measurements & Mean & Left knee & Right knee & Male & Female \\
\hline Hamstring diameter $(\mathrm{mm})$ & $7.01 \pm 0.64$ & $6.82 \pm 0.74$ & $7.17 \pm 0.54$ & $7.45 \pm 0.3$ & $6.34 \pm 0.33$ \\
Hamstring mid area $\left(\mathrm{mm}^{2}\right)$ & $38.02 \pm 6.83$ & $37.49 \pm 7.14$ & $38.49 \pm 7.01$ & $43.35 \pm 1.32$ & $30.03 \pm 0.34$ \\
ACL mid diameter $(\mathrm{mm})$ & $6.2 \pm 0.59$ & $5.98 \pm 0.63$ & $6.39 \pm 0.5$ & $6.6 \pm 0.27$ & $5.59 \pm 0.34$ \\
ACL mid area $\left(\mathrm{mm}^{2}\right)$ & $29.69 \pm 3.87$ & $28.86 \pm 4.43$ & $30.42 \pm 3.44$ & $32.57 \pm 1.35$ & $25.37 \pm 1.31$ \\
PCL mid diameter $(\mathrm{mm})$ & $7.16 \pm 0.7$ & $6.91 \pm 0.76$ & $7.39 \pm 0.61$ & $7.65 \pm 0.31$ & $6.43 \pm 0.39$ \\
PCL mid area $\left(\mathrm{mm}^{2}\right)$ & $34.28 \pm 4.64$ & $33 \pm 5.03$ & $35.4 \pm 4.28$ & $37.72 \pm 1.53$ & $29.12 \pm 1.77$ \\
\hline ACL-tibial insertion length $(\mathrm{mm})$ & $13.75 \pm 1.21$ & $13.59 \pm 1.21$ & $13.9 \pm 1.28$ & $14.58 \pm 0.79$ & $12.51 \pm 0.14$ \\
ACL-tibial insertion width $(\mathrm{mm})$ & $10.74 \pm 0.77$ & $10.62 \pm 0.76$ & $10.84 \pm 0.82$ & $11.23 \pm 0.61$ & $10.01 \pm 0.11$ \\
ACL-femoral insertion length $(\mathrm{mm})$ & $13.21 \pm 1.19$ & $13.05 \pm 1.19$ & $13.36 \pm 1.24$ & $14.03 \pm 0.76$ & $11.99 \pm 0.14$ \\
ACL-femoral insertion width $(\mathrm{mm})$ & $9.37 \pm 1.33$ & $9.72 \pm 1.54$ & $9.06 \pm 1.14$ & $10.05 \pm 1.3$ & $8.34 \pm 0.4$ \\
ACL-tibial footprint area $\left(\mathrm{mm}{ }^{2}\right)$ & $118.2 \pm 18.43$ & $122.64 \pm 21.5$ & $114.31 \pm 15.69$ & $128.6 \pm 16.6$ & $102.6 \pm 4.86$ \\
ACL-femoral footprint area $\left(\mathrm{mm}^{2}\right)$ & $99.6 \pm 19.41$ & $103.53 \pm 22.27$ & $96.16 \pm 17.31$ & $112.2 \pm 14.43$ & $80.7 \pm 2.75$ \\
\hline PCL-tibial insertion length $\left(\mathrm{mm}^{2}\right)$ & $15.9 \pm 1.46$ & $15.71 \pm 1.47$ & $16.07 \pm 1.54$ & $16.91 \pm 0.92$ & $14.38 \pm 0.16$ \\
PCL-tibial insertion width $\left(\mathrm{mm}^{2}\right)$ & $12.55 \pm 1.04$ & $12.4 \pm 1.03$ & $12.67 \pm 1.1$ & $13.24 \pm 0.72$ & $11.51 \pm 0.13$ \\
PCL-femoral insertion length $(\mathrm{mm})$ & $15.23 \pm 1.48$ & $15.03 \pm 1.5$ & $15.4 \pm 1.55$ & $16.27 \pm 0.89$ & $13.67 \pm 0.16$ \\
PCL-femoral insertion width $(\mathrm{mm})$ & $11.12 \pm 1.81$ & $11.44 \pm 2.07$ & $10.8 \pm 1.6$ & $12.08 \pm 1.53$ & $9.4 \pm 0.42$ \\
PCL-tibial footprint area $\left(\mathrm{mm}^{2}\right)$ & $135.26 \pm 21.13$ & $141.8 \pm 25.38$ & $129.54 \pm 16.13$ & $146.78 \pm 19.71$ & $117.98 \pm 5.58$ \\
PCL-femoral footprint area $\left(\mathrm{mm}^{2}\right)$ & $120.69 \pm 29.17$ & $121.09 \pm 27.47$ & $120.35 \pm 32.48$ & $139.3 \pm 22.56$ & $92.78 \pm 3.15$ \\
\hline
\end{tabular}

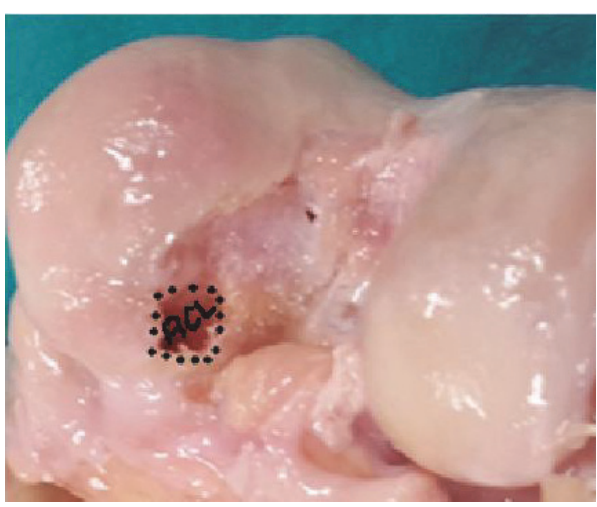

(a)

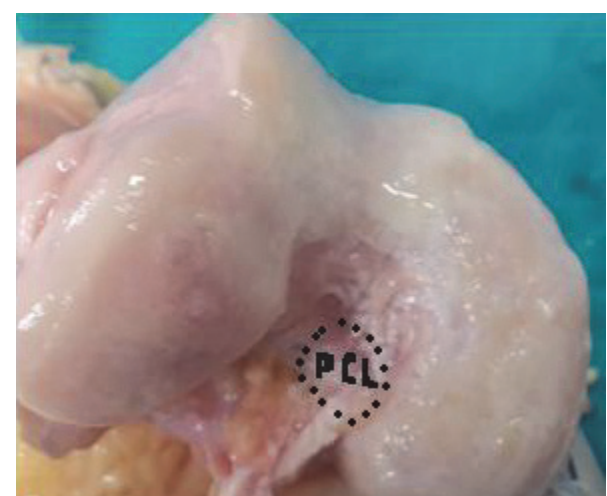

(b)

FIgURE 3: Measurements of the surface area of the femoral entry area without the synovial membrane.

tendons was found to be significantly larger than the diameter of the ACL in the midsection (mean 13.06\%). A positive statistically significant relationship at $96.2 \%$ was determined between the ACL mid diameters and the hamstring diameters $(p<0.01)$. The mean cross-section area of the hamstring tendons was found to be significantly larger than the area of the ACL in the midsection (mean 28.05\%). A positive statistically significant relationship at $96.7 \%$ was determined between the ACL mid areas and the hamstring mid areas $(p<0.01)$.

The diameter of the hamstring tendons was significantly smaller than the PCL diameter in the midsection (mean $2.13 \%)$. A positive statistically significant relationship at 96.4\% was determined between the PCL mid diameters and the hamstring diameters $(p<0.01)$. The cross-section area of the hamstring tendons was significantly greater than the PCL area in the midsection (mean $10.91 \%$ ). A positive statistically significant relationship at $95.7 \%$ was determined between the PCL mid areas and the hamstring mid areas $(p<0.01)$.

The dimensions of the hamstring tendons, ACL, and PCL were determined to be smaller in females than in males. The statistically significant positive relationship between ACL mid diameters and hamstring diameters was higher in females $(89.1 \%, p=0.011)$ than in males $(79.8 \%, p=0.017)$ $(p<0.05)$. Similarly, the statistically significant positive relationship between PCL mid diameters and hamstring diameters was higher in females $(89.1 \%, p=0.011)$ than in males $(79.5 \%, p=0.017)(p<0.05)$.

\section{Discussion}

The most significant finding of this study was that, despite the good correlation between the midsection cross-sectional area of both ACL and PCL, for the reconstruction of these 
TABLE 2: Correlations of the hamstring diameter and areas with the ACL and PCL diameter and areas.

\begin{tabular}{lcc}
\hline & $r$ & $p$ \\
\hline ACL mid diameter $(\mathrm{mm})$ & & \\
Mean & 0.962 & $0.001^{* *}$ \\
Left knee & 0.983 & $0.001^{* *}$ \\
Right knee & 0.933 & $0.001^{* *}$ \\
Male & 0.798 & $0.011^{*}$ \\
Female & 0.891 & $0.017^{*}$ \\
\hline PCL mid diameter $(\mathrm{mm})$ & & \\
Mean & 0.964 & $0.001^{* *}$ \\
Left knee & 0.984 & $0.001^{* *}$ \\
Right knee & 0.933 & $0.001^{* *}$ \\
Male & 0.795 & $0.011^{*}$ \\
Female & 0.891 & $0.017^{*}$ \\
\hline ACL mid area $\left(\mathrm{mm}^{2}\right)$ & & \\
Mean & 0.967 & $0.001^{* *}$ \\
Left knee & 0.980 & $0.001^{* *}$ \\
Right knee & 0.985 & $0.001^{* *}$ \\
Male & 0.801 & $0.011^{*}$ \\
Female & 0.630 & 0.180 \\
\hline PCL mid area $\left(\mathrm{mm}^{2}\right)$ & & \\
Mean & 0.957 & $0.001^{* *}$ \\
Left knee & 0.978 & $0.001^{* *}$ \\
Right knee & 0.976 & $0.001^{* *}$ \\
Male & 0.608 & 0.083 \\
Female & 0.583 & \\
\hline
\end{tabular}

Pearson correlation analysis ${ }^{*} p<0.05{ }^{* *} p<0.01$.

ligaments, there was determined to be less incompatibility between the four-strand hamstring tendons taken from the same knee and the dimensions of the midsection PCL compared to the ACL dimensions. Such a correlation for the PCL in particular is revealed for the first time in this study.

Injuries of the knee ligaments have been reported at the rate of 60.9 per 100,000 people and ACL injuries are seen more than PCL injuries $[10,11]$. The general approach in surgery for the incapacity created by these ligament injuries is intra-articular anatomic reconstruction of the ligaments rather than primary repair. However, there is as yet no consensus on subjects such as graft selection, placement, and fixation in both ACL and PCL reconstruction [12-14]. In addition, graft selection depends on the surgeon's experience, preference, tissue status, the age and activity level of the patient, concomitant diseases, the preoperative status, and the patient's wishes $[12,15,16]$. Hamstring tendons are known to still be the most commonly used grafts in ACL and PCL reconstruction $[17,18]$. However, the correlations of ACL and PCL dimensions with hamstring grafts have a very small place in recent literature [6].

In comparison with other cadaver studies which have defined ACL and PCL dimensions, the mean age of the cadavers, the mean BMI measurements, and the waiting time of the cadavers on which the measurements were made to evaluate the correlations have been reported to be similar to those of the current study [6]. While some researchers have used a micrometer or digital calipers to manually measure the width and length for the evaluation of this correlation, others have used different methods such as digital picture analysis, radiographs, or MRI [19-22]. In the current study, measurements were made manually using digital calipers. Therefore, the results of this study can be considered to be more sensitive and more reliable.

There are various measurement techniques with different results for ACL and PCL [23-25]. Different results have been obtained in particular when measurements have been made before and after the removal of synovial membrane [26]. This was taken into consideration for the current study and in the belief of being able to make a more accurate correlation evaluation, cleaning of the synovial membrane was preferred. The results obtained were seen to be similar to the results obtained in other studies where the synovial membrane was removed [27-29].

When anatomic placement of the ligaments was examined, it was seen that the ACL is wider in the entry area and has a biconcave shape with the narrowest part in the midsection. The width in the entry area is generally in the anteroposterior direction. In other words, the midsection of the ligament is approximately 3.5 times smaller than the ACL projection [22]. Similarly, when the PCL was examined, the tibial attachment point of the ligament is more inferior and posterior compared to the tibial joint surface and is a trapezoidal area. The femoral attachment point is anteromedial to the intercondylar notch wall when the knee is in extension. With this structure, one of the most important ligaments providing stability of the knee joint is the PCL, the main function of which is to prevent posterior translation of the tibia below the femur. A secondary function is to aid rotational and varus-valgus stability of the knee. If it is considered that the tensile strength of the PCL is approximately twice that of the ACL, it is accepted as the primary stabiliser of the knee $[29,30]$. Due to this structure, the PCL has always been evaluated within all the knee ligaments as the "central pivot" [31]. Therefore, in the last 10 years there have been studies which have reported improvements in functional results after isolated PCL reconstruction and indicated the potential benefits of surgery in certain cases [32].

Although the anatomic structure of both the ACL and PCL is well understood, the question arises of how compatible hamstring tendons are, which are currently often used as the most appropriate graft, in place of these ligaments. The compatibility of hamstring grafts with both ACL and PCL may vary according to gender and the individual's body structure. While extremely intense debate on surgical reconstruction techniques continues, the use of sufficiently robust grafts is recommended for both ligaments. Second-look arthroscopic examinations have shown that generally deterioration of the graft has occurred in the midsection $[8,9,33]$. One condition which could cause this is the compatibility of the graft used for reconstruction in place of both ACL and PCL. Studies in literature showing that it is not correct to think that, as the graft thickness increases, stability increases have brought this consideration to the fore $[7,34]$. 
It is well known that the anatomic restoration of native foot print anatomy in ACLR is of paramount importance regardless of graft size and source. However, oversizing of the graft has a negative effect on the healing process and kinematics [6]. Therefore, the goals of ACL/PCL reconstruction should be the anatomic reconstruction of foot prints with an appropriate size of graft which should match the native ACL/PCL. In this study, both ACL/PCL cross-sectional area measurements were found to match with four-strand hamstring tendon grafts. However, the particular contribution of this study to literature is that PCL midsubstance crosssectional area measurements matched the four-strand hamstring tendon grafts, and this point has not been previously studied.

Together with these evaluations, there were some limitations to this study, primarily the number of cadavers and the fact that the mean age of the cadavers was significantly higher than that of most patients who undergo ACL and PCL reconstruction. Even if the number of cadavers can be increased with further studies, the advanced age of the cadavers could affect the structure and morphology of the ACL and PCL. Furthermore, the cross-sectional geometric forms of both the ACL and PCL were found to be irregular in that they were not purely circular or purely elliptical. Therefore, the calculations can only reflect approximate results, not exact results [12].

\section{Conclusion}

Compared with current ACL reconstruction techniques, grafting has a greater diameter despite the good correlation between four-strand hamstring tendons and the midsection cross-sectional areas, especially of the ACL. Moreover, compared with current PCL reconstruction techniques, there is a good correlation between four-strand hamstring tendons and the midsection cross-sectional areas in particular of the PCL. Therefore, compared to ACL reconstruction, in the anatomic reconstruction of $\mathrm{PCL}$, four-strand hamstring tendons could be more compatible with the footprint of these ligaments or could be considered to mimic the midsection of the ligament.

\section{Competing Interests}

The authors declare that they have no competing interests.

\section{Authors' Contributions}

Barış Yılmaz, Güzelali Özdemir, Elif N. Keskinöz, Gamze Tümentemur, Kemal Gökkuş, and Bahtiyar Demiralp designed the study, collected data, assessed the results, and wrote the manuscript.

\section{References}

[1] T. Zantop, W. Petersen, J. K. Sekiya, V. Musahl, and F. H. Fu, "Anterior cruciate ligament anatomy and function relating to anatomical reconstruction," Knee Surgery, Sports Traumatology, Arthroscopy, vol. 14, no. 10, pp. 982-992, 2006.
[2] D. K. Cho, S. P. Rosa, G. B. Prestes, L. A. M. da Cunha, M. F. A. de Moura, and E. S. Filho, "Anatomical study of the posterior cruciate ligament with the knee flexed at $90^{\circ}$, " Revista Brasileira de Ortopedia, vol. 49, no. 5, pp. 494-498, 2014.

[3] A. Kaya, M. Köken, B. Akan, D. Karagüven, and B. Güçlü, "The triangle between the anterior and posterior cruciate ligaments: an arthroscopic anatomy study," Acta Orthopaedica et Traumatologica Turcica, vol. 49, no. 5, pp. 478-482, 2015.

[4] Z. Taşdemir, D. Gülabi, F. Sağlam, S. T. Özal, and N. Elmalı, "Does the anteromedial portal provide clinical superiority compared to the transtibial portal in anterior cruciate ligament reconstruction in nonprofessional athletes in short-term follow-up?" Acta Orthopaedica et Traumatologica Turcica, vol. 49, no. 5, pp. 483-491, 2015.

[5] J. P. Sullivan, S. Cook, Y. Gao, and B. R. Wolf, "Radiographic anatomy of the native anterior cruciate ligament: a systematic review," HSS Journal, vol. 11, no. 2, pp. 154-165, 2015.

[6] N. Pujol, S. Queinnec, P. Boisrenoult, A. Maqdes, and P. Beaufils, "Anatomy of the anterior cruciate ligament related to hamstring tendon grafts. A cadaveric study," Knee, vol. 20, no. 6, pp. 511514, 2013.

[7] A. Bedi, T. Maak, V. Musahl et al., "Effect of tunnel position and graft size in single-bundle anterior cruciate ligament reconstruction: an evaluation of time-zero knee stability," Arthroscopy, vol. 27, no. 11, pp. 1543-1551, 2011.

[8] J. M. Marzo, M. K. Bowen, R. F. Warren, T. L. Wickiewicz, and D. W. Altchek, "Intraarticular fibrous nodule as a cause of loss of extension following anterior cruciate ligament reconstruction," Arthroscopy: The Journal of Arthroscopic and Related Surgery, vol. 8, no. 1, pp. 10-18, 1992.

[9] Y. Toritsuka, K. Shino, S. Horibe et al., "Second-look arthroscopy of anterior cruciate ligament grafts with multistranded hamstring tendons," Arthroscopy, vol. 20, no. 3, pp. 287-293, 2004.

[10] J. Parkkari, K. Pasanen, V. M. Mattila, P. Kannus, and A. Rimpelä, “The risk for a cruciate ligament injury of the knee in adolescents and young adults: a population-based cohort study of 46500 people with a 9 year follow-up," British Journal of Sports Medicine, vol. 42, no. 6, pp. 422-426, 2008.

[11] D. M. Swenson, C. L. Collins, T. M. Best, D. C. Flanigan, S. K. Fields, and R. D. Comstock, "Epidemiology of knee injuries among U.S. high school athletes, 2005/2006-2010/2011," Medicine and Science in Sports and Exercise, vol. 45, no. 3, pp. 462-469, 2013.

[12] C. D. Harner, E. Olson, J. J. Irrgang, S. Silverstein, F. H. Fu, and M. Silbey, "Allograft versus autograft anterior cruciate ligament reconstruction: 3- to 5-year outcome," Clinical Orthopaedics and Related Research, vol. 324, pp. 134-144, 1996.

[13] C. Zeng, S.-G. Gao, H. Li et al., "Autograft versus allograft in anterior cruciate ligament reconstruction: a meta-analysis of randomized controlled trials and systematic review of overlapping systematic reviews," Arthroscopy, vol. 32, no. 1, pp. 153.e18163.e18, 2016

[14] C. R. Bottoni, E. L. Smith, J. Shaha et al., "Autograft versus allograft anterior cruciate ligament reconstruction: a prospective, randomized clinical study with a minimum 10 -year follow-up," The American Journal of Sports Medicine, vol. 43, no. 10, pp. 2501-2509, 2015.

[15] R. V. West and C. D. Harner, "Graft selection in anterior cruciate ligament reconstruction," Journal of the American Academy of Orthopaedic Surgeons, vol. 13, no. 3, pp. 197-207, 2005. 
[16] E. Romanini, F. D’Angelo, S. De Masi et al., "Graft selection in arthroscopic anterior cruciate ligament reconstruction," Journal of Orthopaedics and Traumatology, vol. 11, no. 4, pp. 211-219, 2010.

[17] F. R. Noyes, D. L. Butler, E. S. Grood, R. F. Zernicke, and M. S. Hefzy, "Biomechanical analysis of human ligament grafts used in knee-ligament repairs and reconstructions," Journal of Bone and Joint Surgery-Series A, vol. 66, no. 3, pp. 344-352, 1984.

[18] D. L. Hamner, C. H. Brown Jr., M. E. Steiner, A. T. Hecker, and W. C. Hayes, "Hamstring tendon grafts for reconstruction of the anterior cruciate ligament: biomechanical evaluation of the use of multiple strands and tensioning techniques," The Journal of Bone \& Joint Surgery - American Volume, vol. 81, no. 4, pp. 549$557,1999$.

[19] J. Dargel, J. Feiser, M. Gotter, D. Pennig, and J. Koebke, "Side differences in the anatomy of human knee joints," Knee Surgery, Sports Traumatology, Arthroscopy, vol. 17, no. 11, pp. 1368-1376, 2009.

[20] P. Colombet, J. Robinson, P. Christel et al., "Morphology of anterior cruciate ligament attachments for anatomic reconstruction: a cadaveric dissection and radiographic study," Arthroscopy, vol. 22, no. 9, pp. 984-992, 2006.

[21] S. B. Cohen, C. VanBeek, J. S. Starman, D. Armfield, J. J. Irrgang, and F. H. Fu, "MRI measurement of the 2 bundles of the normal anterior cruciate ligament," Orthopedics, vol. 32, no. 9, p. 687, 2009.

[22] C. D. Harner, Goo Hyun Baek, T. M. Vogrin, G. J. Carlin, S. Kashiwaguchi, and S. L.-Y. Woo, "Quantitative analysis of human cruciate ligament insertions," Arthroscopy, vol. 15, no. 7, pp. 741-749, 1999.

[23] S. P. Arnoczky, "Anatomy of the anterior cruciate ligament," Clinical Orthopaedics and Related Research, vol. 172, pp. 19-25, 1983.

[24] S. Kopf, V. Musahl, S. Tashman, M. Szczodry, W. Shen, and F. H. Fu, "A systematic review of the femoral origin and tibial insertion morphology of the ACL," Knee Surgery, Sports Traumatology, Arthroscopy, vol. 17, no. 3, pp. 213-219, 2009.

[25] C. D. Harner, G. A. Livesay, S. Kashiwaguchi, H. Fujie, N. Y. Choi, and S. L. Woo, "Comparative study of the size and shape of human anterior and posterior cruciate ligaments," Journal of Orthopaedic Research, vol. 13, no. 3, pp. 429-434, 1995.

[26] E. K. Bicer, S. Lustig, E. Servien, T. A. S. Selmi, and P. Neyret, "Current knowledge in the anatomy of the human anterior cruciate ligament," Knee Surgery, Sports Traumatology, Arthroscopy, vol. 18, no. 8, pp. 1075-1084, 2010.

[27] R. Siebold, T. Ellert, S. Metz, and J. Metz, “Tibial insertions of the anteromedial and posterolateral bundles of the anterior cruciate ligament: morphometry, arthroscopic landmarks, and orientation model for bone tunnel placement," ArthroscopyJournal of Arthroscopic and Related Surgery, vol. 24, no. 2, pp. 154-161, 2008.

[28] T. Mochizuki, T. Muneta, T. Nagase, S.-I. Shirasawa, K.-I. Akita, and I. Sekiya, "Cadaveric knee observation study for describing anatomic femoral tunnel placement for two-bundle anterior cruciate ligament reconstruction," Arthroscopy, vol. 22, no. 4, pp. 356-361, 2006.

[29] J. Dargel, P. Pohl, P. Tzikaras, and J. Koebke, "Morphometric side-to-side differences in human cruciate ligament insertions," Surgical and Radiologic Anatomy, vol. 28, no. 4, pp. 398-402, 2006.

[30] Y.-M. Kim, C. A. Lee, and M. J. Matava, "Clinical results of arthroscopic single-bundle transtibial posterior cruciate ligament reconstruction: a systematic review," The American Journal of Sports Medicine, vol. 39, no. 2, pp. 425-434, 2011.

[31] K. D. Shelbourne, M. Clark, and T. Gray, "Minimum 10-year follow-up of patients after an acute, isolated posterior cruciate ligament injury treated nonoperatively," The American Journal of Sports Medicine, vol. 41, no. 7, pp. 1526-1533, 2013.

[32] K. D. Shelbourne and T. Gray, "Minimum 10-year results after anterior cruciate ligament reconstruction: how the loss of normal knee motion compounds other factors related to the development of osteoarthritis after surgery," American Journal of Sports Medicine, vol. 37, no. 3, pp. 471-480, 2009.

[33] J. H. Ahn, J. C. Yoo, H. S. Yang, J. H. Kim, and J. H. Wang, "Second-look arthroscopic findings of 208 patients after ACL reconstruction," Knee Surgery, Sports Traumatology, Arthroscopy, vol. 15, no. 3, pp. 242-248, 2007.

[34] E. Triantafyllidi, N. K. Paschos, A. Goussia et al., "The shape and the thickness of the anterior cruciate ligament along its length in relation to the posterior cruciate ligament: a cadaveric study," Arthroscopy, vol. 29, no. 12, pp. 1963-1973, 2013. 


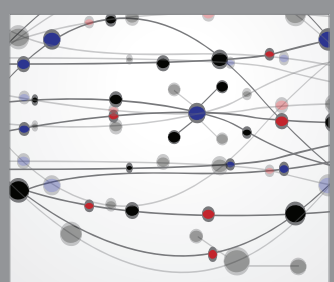

The Scientific World Journal
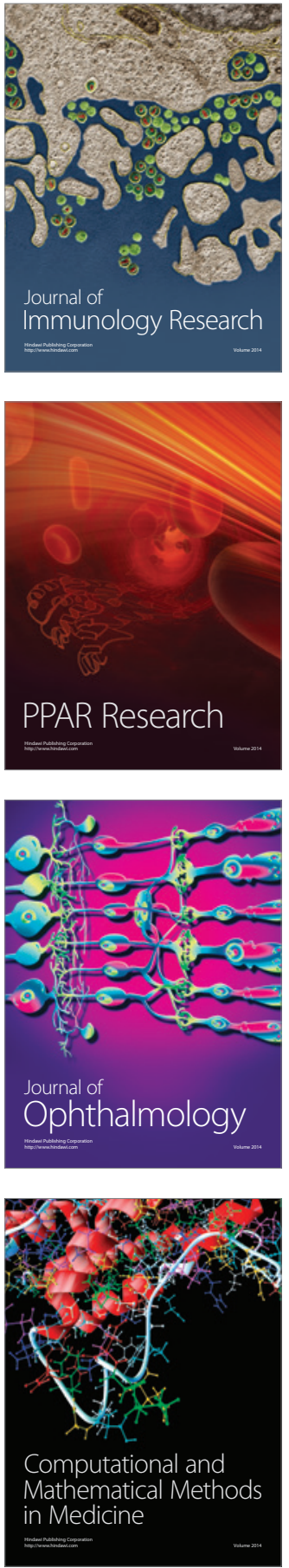

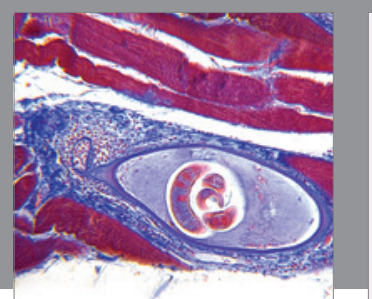

Gastroenterology Research and Practice

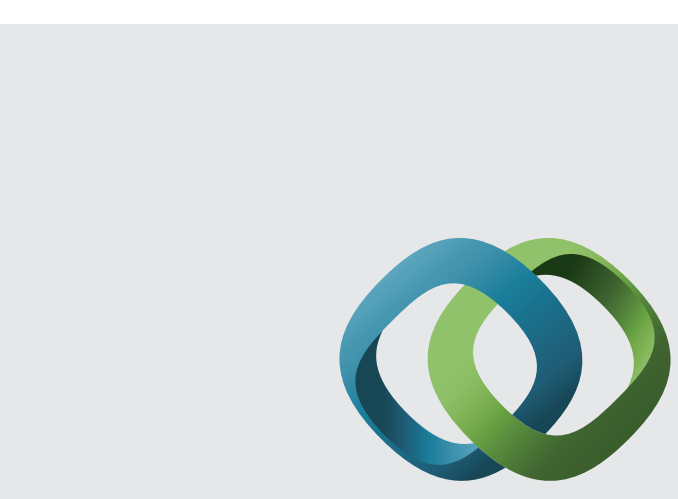

\section{Hindawi}

Submit your manuscripts at

http://www.hindawi.com
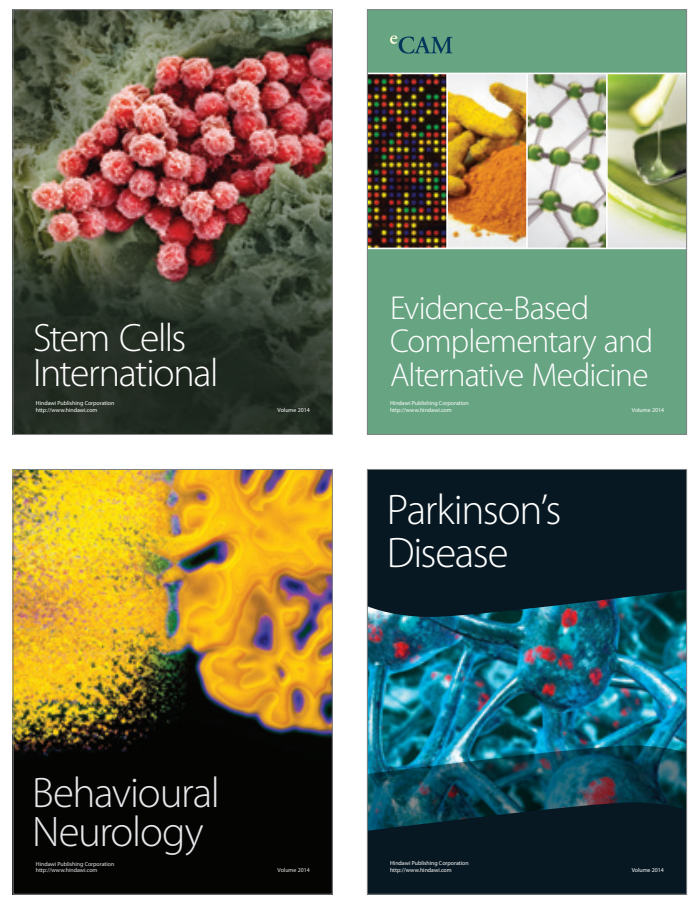
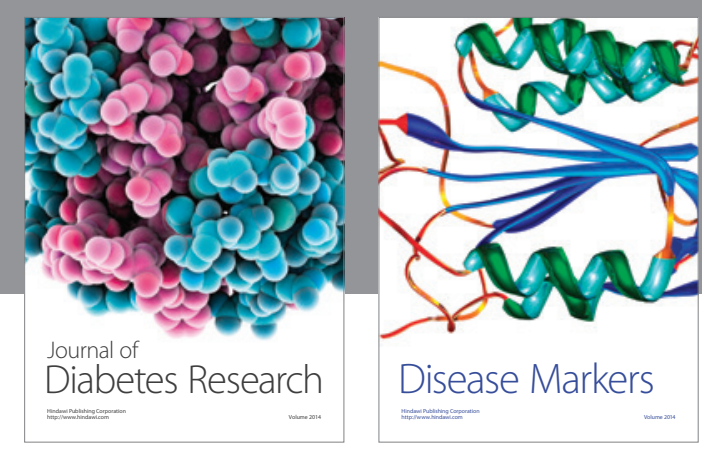

Disease Markers
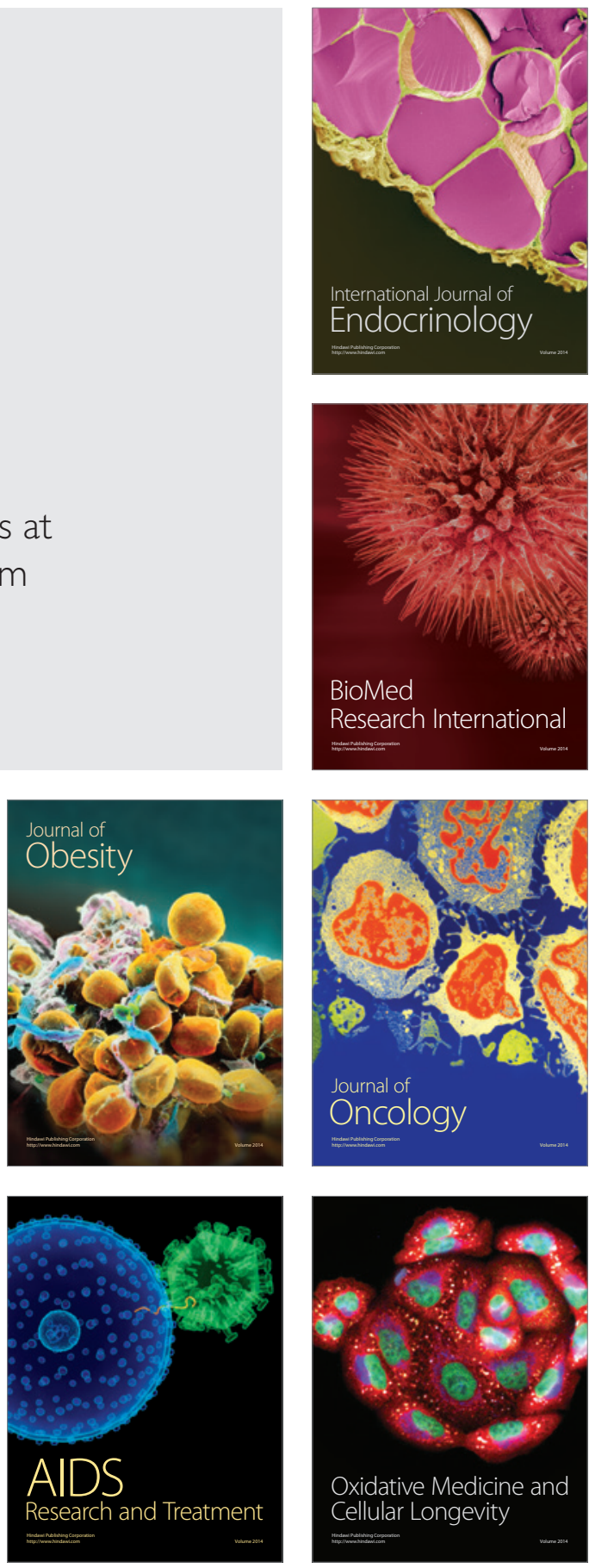